\title{
Free subgroups within the images of quantum representations
}

\author{
Louis Funar \\ Institut Fourier BP 74, UMR 5582 \\ University of Grenoble I \\ 38402 Saint-Martin-d'Hères cedex, France \\ e-mail: funar@fourier.ujf-grenoble.fr
}

\author{
Toshitake Kohno \\ IPMU, Graduate School of Mathematical Sciences \\ The University of Tokyo \\ 3-8-1 Komaba, Meguro-Ku, Tokyo 153-8914 Japan \\ e-mail: kohno@ms.u-tokyo.ac.jp
}

November 21, 2018

\begin{abstract}
We prove that, except for a few explicit roots of unity, the quantum image of any Johnson subgroup of the mapping class group contains an explicit free non-abelian subgroup.

2000 MSC Classification: 57 M 07, 20 F 36, 20 F 38, 57 N 05.

Keywords: Mapping class group, Dehn twist, triangle group, braid group, Burau representation, Johnson filtration, quantum representation.
\end{abstract}

\section{Introduction and statements}

The aim of this paper is to study the images of the mapping class groups by quantum representations. Some results in this direction are already known. We refer the reader to [29] and [19] for earlier treatments of quantum representations. In [8] we proved that the images are infinite and non-abelian (for all but finitely many explicit cases) using earlier results of Jones who proved in [17] that the same holds true for the braid group representations factorizing through the TemperleyLieb algebra at roots of unity. Masbaum then found in [23] explicit elements of infinite order in the image. General arguments concerning Lie groups actually show that the image should contain a free non-abelian group. Furthermore, Larsen and Wang showed (see [21]) that the image of the quantum representations of the mapping class groups at roots of unity of the form $\exp \left(\frac{2 \pi i}{4 r}\right)$, for prime $r \geq 5$, is dense in the projective unitary group.

In order to be precise we have to specify the quantum representations we are considering. Recall that in [2] the authors defined the TQFT functor $\mathcal{V}_{p}$, for every $p \geq 3$ and a primitive root of unity $A$ of order $2 p$. These TQFT should correspond to the so-called $S U(2)$-TQFT, for even $p$ and to the $S O(3)$-TQFT, for odd $p$ (see also [21] for another $S O(3)-\mathrm{TQFT}$ ).

Definition 1.1. Let $p \in \mathbb{Z}_{+}, p \geq 3$, such that $p \not \equiv 2(\bmod 4)$. The quantum representation $\rho_{p}$ is the projective representation of the mapping class group associated to the TQFT $\mathcal{V}_{\frac{p}{2}}$ for even $p$ and $\mathcal{V}_{p}$ for odd $p$, corresponding to the following choices of the root of unity:

$$
A_{p}= \begin{cases}-\exp \left(\frac{2 \pi i}{p}\right), & \text { if } p \equiv 0(\bmod 4) \\ -\exp \left(\frac{(p+1) \pi i}{p}\right), & \text { if } p \equiv 1(\bmod 2)\end{cases}
$$

Notice that $A_{p}$ is a primitive root of unity of order $p$ when $p$ is even and of order $2 p$ otherwise.

Remark 1.1. The eigenvalues of a Dehn twist in the TQFT $\mathcal{V}_{p}$ i.e., the entries of the diagonal $T$-matrix are of the form $\mu_{l}=\left(-A_{p}\right)^{l(l+2)}$, where $l$ belongs to the set of admissible colors (see [2], 
4.11). The set of admissible colors is $\left\{0,1,2, \ldots, \frac{p}{2}-2\right\}$, for even $p$ and is $\{0,2,4, \ldots, p-3\}$ for odd $p$. Therefore the order of the image of a Dehn twist by $\rho_{p}$ is $p$.

We will now consider the Johnson filtration by the subgroups $I_{g}(k)$ of the mapping class group $M_{g}$ of the closed orientable surface of genus $g$, consisting of those elements having a trivial outer action on the $k$-th nilpotent quotient of the fundamental group of the surface, for some $k \in \mathbb{Z}_{+}$. As is well-known the Johnson filtration shows up within the framework of finite type invariants of 3-manifolds (see e.g. [1]).

Our next result shows that the image is large in the following sense (see also Propositions 3.1 and 3.4):

Theorem 1.1. Assume that $g \geq 3$ and $p \notin\{3,4,8,12,16,24\}$ or $g=2, p$ is even and $p \notin$ $\{4,8,12,16,24,40\}$. Then for any $k$, the image $\rho_{p}\left(I_{g}(k)\right)$ of the $k$-th Johnson subgroup by the quantum representation $\rho_{p}$ contains a free non-abelian group.

The idea of proof for this theorem is to embed a pure braid group within the mapping class group and to show that its image is large. Namely, a 4-holed sphere suitably embedded in the surface leads to an embedding of the pure braid group $P B_{3}$ in the mapping class group. The quantum representation contains a particular sub-representation which is the restriction of Burau's representation (see [8]) to a free subgroup of $P B_{3}$. One way to obtain elements of the Johnson filtration is to consider elements of the lower central series of $P B_{3}$ and extend them to all of the surface by identity. Therefore it suffices to find free non-abelian subgroups in the image of the lower central series of $P B_{3}$ by Burau's representation at roots of unity in order to prove Theorem 1.1 .

The analysis of the contribution of mapping classes supported on small sub-surfaces of a surface, which are usually holed spheres, to various subgroups of the mapping class groups was also used in an unpublished paper by T. Oda and J. Levine (see [22]) for obtaining lower bounds for the ranks of the graded quotients of the Johnson filtration.

Our construction also provides explicit free non-abelian subgroups (see Theorems 3.1 and 3.2 for precise statements).

Acknowledgements. We are grateful to Jørgen Andersen, Greg Kuperberg, Greg McShane and Gregor Masbaum for useful discussions and to Ian Agol for pointing out a gap in the previous version of this paper. The second author is partially supported by Grant-in-Aid for Scientific Research 23340014, Japan Society for Promotion of Science, and by World Premier International Research Center Initiative, MEXT, Japan. A part of this work was accomplished while the second author was staying at Institut Fourier in Grenoble. He would like to thank Institut Fourier for hospitality.

\section{Burau's representations of $B_{3}$ and triangle groups}

Let $B_{n}$ denote the braid group on $n$ strands with the standard generators $g_{1}, g_{2}, \ldots, g_{n-1}$. Squier was interested to compare the kernel of Burau's representation $\beta_{q}$ at a $k$-th root of unity $q$ with the normal subgroup $B_{n}[k]$ of $B_{n}$ generated by $g_{j}^{k}, 1 \leq j \leq n-1$. Recall that:

Definition 2.1. The (reduced) Burau representation $\beta: B_{n} \rightarrow G L\left(n-1, \mathbb{Z}\left[q, q^{-1}\right]\right)$ is defined on the standard generators

$$
\beta_{q}\left(g_{1}\right)=\left(\begin{array}{cc}
-q & 1 \\
0 & 1
\end{array}\right) \oplus \mathbf{1}_{n-3},
$$




$$
\begin{gathered}
\beta_{q}\left(g_{j}\right)=\mathbf{1}_{j-2} \oplus\left(\begin{array}{ccc}
1 & 0 & 0 \\
q & -q & 1 \\
0 & 0 & 1
\end{array}\right) \oplus \mathbf{1}_{n-j-2}, \text { for } 2 \leq j \leq n-2, \\
\beta_{q}\left(g_{n-1}\right)=\mathbf{1}_{n-3} \oplus\left(\begin{array}{cc}
1 & 0 \\
q & -q
\end{array}\right) .
\end{gathered}
$$

The paper [10] is devoted to the complete description of the image of Burau's representation of $B_{3}$ at roots of unity. Similar results were obtained in [20, 23, 25]. For the sake of completeness we review here the essential tools from [10] to be used later.

Let us denote by $A=\beta_{-q}\left(g_{1}^{2}\right)$ and $B=\beta_{-q}\left(g_{2}^{2}\right)$ and $C=\beta_{-q}\left(\left(g_{1} g_{2}\right)^{3}\right)$. As is well-known $P B_{3}$ is isomorphic to the direct product $\mathbb{F}_{2} \times \mathbb{Z}$, where $\mathbb{F}_{2}$ is freely generated by $g_{1}^{2}$ and $g_{2}^{2}$ and the factor $\mathbb{Z}$ is the center of $B_{3}$ generated by $\left(g_{1} g_{2}\right)^{3}$.

It is simple to check that:

$$
A=\left(\begin{array}{cc}
q^{2} & 1+q \\
0 & 1
\end{array}\right), B=\left(\begin{array}{cc}
1 & 0 \\
-q-q^{2} & q^{2}
\end{array}\right), C=\left(\begin{array}{cc}
-q^{3} & 0 \\
0 & -q^{3}
\end{array}\right)
$$

Recall that $P S L(2, \mathbb{Z})$ is the quotient of $B_{3}$ by its center. Since $C$ is a scalar matrix the homomorphism $\beta_{-q}: B_{3} \rightarrow G L(2, \mathbb{C})$ factors to a homomorphism $P S L(2, \mathbb{Z}) \rightarrow P G L(2, \mathbb{C})$.

We will be concerned below with the subgroup $\Gamma_{-q}$ of $P G L(2, \mathbb{C})$ generated by the images of $A$ and $B$ in $P G L(2, \mathbb{C})$. When $\beta_{-q}$ is unitarizable, the group $\Gamma_{-q}$ can be viewed as a subgroup of the complex-unitary group $P U(1,1)$.

Before we proceed we make a short digression on triangle groups. Let $\Delta$ be a geodesic triangle in the hyperbolic plane of angles $\frac{\pi}{m}, \frac{\pi}{n}, \frac{\pi}{p}$, so that $\frac{1}{m}+\frac{1}{n}+\frac{1}{p}<1$. The extended triangle group $\Delta^{*}(m, n, p)$ is the group of isometries of the hyperbolic plane generated by the three reflections $R_{1}, R_{2}, R_{3}$ with respect to the edges of $\Delta$. It is well-known that a presentation of $\Delta^{*}(m, n, p)$ is given by

$$
\Delta^{*}(m, n, p)=\left\langle R_{1}, R_{2}, R_{3} ; R_{1}^{2}=R_{2}^{2}=R_{3}^{2}=1,\left(R_{1} R_{2}\right)^{m}=\left(R_{2} R_{3}\right)^{n}=\left(R_{3} R_{1}\right)^{p}=1\right\rangle .
$$

The second type of relations have a simple geometric meaning. In fact, the product of the reflections with respect to two adjacent edges is a rotation by the angle which is twice the angle between those edges. The subgroup $\Delta(m, n, p)$ generated by the rotations $a=R_{1} R_{2}, b=R_{2} R_{3}, c=R_{3} R_{1}$ is a normal subgroup of index 2 , which coincides with the subgroup of isometries preserving the orientation. One calls $\Delta(m, n, p)$ the triangle (also called triangular, or von Dyck) group associated to $\Delta$. Moreover, the triangle group has the presentation:

$$
\Delta(m, n, p)=\left\langle a, b, c ; a^{m}=b^{n}=c^{p}=1, a b c=1\right\rangle .
$$

Observe that $\Delta(m, n, p)$ also makes sense when $m, n$ or $p$ are negative integers, by interpreting the associated generators as clockwise rotations. The triangle $\Delta$ is a fundamental domain for the action of $\Delta^{*}(m, n, p)$ on the hyperbolic plane. Thus a fundamental domain for $\Delta(m, n, p)$ consists of the union $\Delta^{*}$ of $\Delta$ with the reflection of $\Delta$ in one of its edges.

Proposition 2.1 ([10]). Let $m<k$ be such that $\operatorname{gcd}(m, k)=1$ where $k \geq 4$. Then the group $\Gamma_{-\exp \left(\frac{ \pm 2 m \pi i}{2 k}\right)}$ is a triangle group with the presentation:

$$
\Gamma_{-\exp \left(\frac{ \pm 2 m \pi i}{2 k}\right)}=\left\langle A, B ; A^{k}=B^{k}=(A B)^{k}=1\right\rangle .
$$


If $n$ is odd $n=2 k+1$, then the group $\Gamma_{-q}$ is a quotient of the triangle group associated to $\Delta$, which embeds into the group associated to some sub-triangle $\Delta^{\prime}$ of $\Delta$.

Proposition $2.2([10])$. Let $0<m<2 k+1$ be such that $\operatorname{gcd}(m, 2 k+1)=1$ and $k \geq 3$. Then the group $\Gamma_{-\exp \left(\frac{ \pm 2 m \pi i}{2 k+1}\right)}$ is isomorphic to the triangle group $\Delta(2,3,2 k+1)$ and has the following presentation (in terms of our generators $A, B$ ):

$$
\Gamma_{-\exp \left(\frac{ \pm 2 m \pi i}{2 k+1}\right)}=\left\langle A, B ; A^{2 k+1}=B^{2 k+1}=(A B)^{2 k+1}=1,\left(A^{-1} B^{k}\right)^{2}=1,\left(B^{k} A^{k-1}\right)^{3}=1\right\rangle .
$$

Proof. Here is a sketch of the proof. Deraux proved in ([5], Theorem 7.1) that the group $\Delta\left(\frac{2 k+1}{2}, \frac{2 k+1}{2}, \frac{2 k+1}{2}\right)$, which is generated by the rotations $a, b, c$ around the vertices of the triangle $\Delta$ embeds into the triangle group associated to a smaller triangle $\Delta^{\prime}$. One constructs $\Delta^{\prime}$ by considering all geodesics of $\Delta$ joining a vertex and the midpoint of its opposite side. The three median geodesics pass through the barycenter of $\Delta$ and subdivide $\Delta$ into 6 equal triangles. We can take for $\Delta^{\prime}$ any one of the 6 triangles of the subdivision. It is immediate that $\Delta^{\prime}$ has angles $\frac{\pi}{2 k+1}, \frac{\pi}{2}$ and $\frac{\pi}{3}$ so that the associated triangle group is $\Delta(2,3,2 k+1)$. This group has the presentation:

$$
\Delta(2,3,2 k+1)=\left\langle\alpha, u, v ; \alpha^{2 k+1}=u^{3}=v^{2}=\alpha u v=1\right\rangle,
$$

where the generators are the rotations of double angle around the vertices of the triangle $\Delta^{\prime}$.

Lemma 2.1. The natural embedding of $\Delta\left(\frac{2 k+1}{2}, \frac{2 k+1}{2}, \frac{2 k+1}{2}\right)$ into $\Delta(2,3,2 k+1)$ is an isomorphism.

Proof. A simple geometric computation shows that:

$$
a=\alpha^{2}, b=v \alpha^{2} v=u^{2} \alpha^{2} u, c=u \alpha^{2} u^{2} .
$$

Therefore $\alpha=a^{k+1} \in \Delta\left(\frac{2 k+1}{2}, \frac{2 k+1}{2}, \frac{2 k+1}{2}\right)$.

From the relation $\alpha u v=1$ we derive $a^{k+1} u v=1$, and thus $u=a^{k} v$. The relation $u^{3}=1$ reads now $a^{k}\left(v a^{k} v\right) a^{k} v=1$ and replacing $b^{k}$ by $v a^{k} v$ we find that $v=a^{k} b^{k} a^{k} \in \Delta\left(\frac{2 k+1}{2}, \frac{2 k+1}{2}, \frac{2 k+1}{2}\right)$.

Further $u=a^{k} v=a^{-1} b^{k} a^{k} \in \Delta\left(\frac{2 k+1}{2}, \frac{2 k+1}{2}, \frac{2 k+1}{2}\right)$. This means that $\Delta\left(\frac{2 k+1}{2}, \frac{2 k+1}{2}, \frac{2 k+1}{2}\right)$ is actually $\Delta(2,3,2 k+1)$, as claimed.

It suffices now to find a presentation of $\Delta(2,3,2 k+1)$ that uses the generators $A=a, B=b$. It is not difficult to show that the group with the presentation of the statement is isomorphic to $\Delta(2,3,2 k+1)$, the inverse homomorphism sending $\alpha$ into $A^{k+1}, u$ into $A^{-1} B^{k} A^{k}$ and $v$ into $A^{k} B^{k} A^{k}$.

A direct consequence of Propositions 2.1 and 2.2 is the following abstract description of the image of Burau's representation:

Corollary 2.1. If $q$ is not a primitive root of unity of order in the set $\{1,2,3,4,6,10\}$, then $\Gamma_{q}$ is an infinite triangle group.

Alternatively, we obtain a set of normal generators for the kernel of Burau's representation, as follows:

Corollary 2.2. Let $n \notin\{1,6\}$ and $q$ a primitive root of unity of order $n$. We denote by $N(G)$ the normal closure of a subgroup $G$ of $\left\langle g_{1}^{2}, g_{2}^{2}\right\rangle$. Then the kernel $\operatorname{ker} \beta_{-q}:\left\langle g_{1}^{2}, g_{2}^{2}\right\rangle \rightarrow P G L(2, \mathbb{C})$ of the restriction of Burau's representation is given by:

$$
\begin{cases}N\left(\left\langle g_{1}^{2 k}, g_{2}^{2 k},\left(g_{1}^{2} g_{2}^{2}\right)^{k}\right\rangle\right), & \text { if } n=2 k ; \\ N\left(\left\langle g_{1}^{2(2 k+1)}, g_{2}^{2(2 k+1)},\left(g_{1}^{2} g_{2}^{2}\right)^{2 k+1},\left(g_{1}^{-2} g_{2}^{2 k}\right)^{2},\left(g_{2}^{2 k} g_{1}^{2(k-1)}\right)^{3}\right\rangle\right), & \text { if } n=2 k+1 .\end{cases}
$$




\section{Johnson subgroups and proof of Theorem 1.1}

\subsection{Proof of Theorem 1.1}

For a group $G$ we denote by $G_{(k)}$ the lower central series defined by:

$$
G_{(1)}=G, G_{(k+1)}=\left[G, G_{(k)}\right], k \geq 1
$$

An interesting family of subgroups of the mapping class group is the set of higher Johnson subgroups defined as follows.

Definition 3.1. The $k$-th Johnson subgroup $I_{g}(k)$ is the group of mapping classes of homeomorphisms of the closed orientable surface $\Sigma_{g}$ whose action by outer automorphisms on $\pi / \pi_{(k+1)}$ is trivial, where $\pi=\pi_{1}\left(\Sigma_{g}\right)$.

Thus $I_{g}(0)=M_{g}, I_{g}(1)$ is the Torelli group commonly denoted $T_{g}$, while $I_{g}(2)$ is the group generated by the Dehn twists along separating simple closed curves and considered by Johnson and Morita (see e.g. [16, 26]), which is often denoted by $K_{g}$.

The proof of Theorem 1.1 follows from the same argument as in [8, where we proved that the image of the quantum representation $\rho_{p}$ is infinite for all $p$ in the given range. The values of $p$ which are excluded correspond to the TQFT's $\mathcal{V}_{3}, \mathcal{V}_{2}, \mathcal{V}_{4}, \mathcal{V}_{6}, \mathcal{V}_{8}$ and $\mathcal{V}_{12}$ and it is known that the images of quantum representations are finite in these cases.

Before we proceed we have to make the cautionary remark that $\rho_{p}$ is only a projective representation. Here and henceforth when speaking about Burau's representation we will mean the representation $\beta_{q}: B_{3} \rightarrow P G L(2, \mathbb{C})$ taking values in matrices modulo scalars.

We will first consider the generic case where the genus is large and the 10-th roots of unity are discarded. This will prove Theorem 1.1 in most cases. Specifically we will prove first:

Proposition 3.1. Assume that $g \geq 4$. Then the image $\rho_{p}\left(\left(\left\langle g_{1}^{2}, g_{2}^{2}\right\rangle\right)_{(k)}\right)$ contains a free non-abelian group for every $k$ and $p \notin\{3,4,8,12,16,24,40\}$.

Proof. The first step of the proof provides us with enough elements of $I_{g}(k)$ having their support contained in a small subsurface of $\Sigma_{g}$.

Specifically we embed $\Sigma_{0,4}$ into $\Sigma_{g}$ by means of curves $c_{1}, c_{2}, c_{3}, c_{4}$ as in the figure below. Then the curves $a$ and $b$ which are surrounding two of the holes of $\Sigma_{0,4}$ are separating.

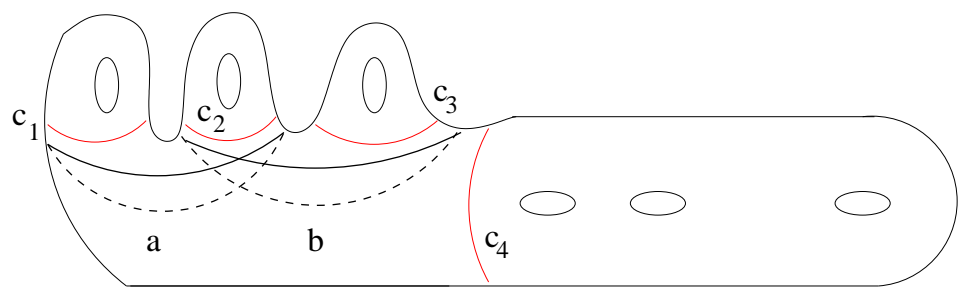

The pure braid group $P B_{3}$ embeds into $M_{0,4}$ using a non-canonical splitting of the surjection $M_{0,4} \rightarrow P B_{3}$. Furthermore, $M_{0,4}$ embeds into $M_{g}$ when $g \geq 4$, by using the homomorphism induced by the inclusion of $\Sigma_{0,4}$ into $\Sigma_{g}$ as in the figure. Then the group generated by the Dehn twists $a$ and $b$ is identified with the free subgroup generated by $g_{1}^{2}$ and $g_{2}^{2}$ into $P B_{3}$. Moreover, $P B_{3}$ has a natural action on a a subspace of the space of conformal blocks associated to $\Sigma_{g}$ as in [8], which is isomorphic to the restriction of Burau's representation at some root of unity depending on $p$. Notice that the two Dehn twists above are elements of $K_{g}$.

We will need the following Proposition whose proof will be given in section 3.1.1 
Proposition 3.2. The above embedding of $P B_{3}$ into $M_{g}$ sends $\left(P B_{3}\right)_{(k)}$ into $I_{g}(k)$.

Recall now that $\left\langle g_{1}^{2}, g_{2}^{2}\right\rangle$ is a normal free subgroup of $P B_{3}$. The second ingredient needed in the proof of Proposition 3.1 is the following Proposition which will be proved in 3.1.2;

Proposition 3.3. Assume that $g \geq 4$. Then the image $\rho_{p}\left(\left(\left\langle g_{1}^{2}, g_{2}^{2}\right\rangle\right)_{(k)}\right)$ contains a free non-abelian group for every $k$ and $p \notin\{3,4,8,12,16,24,40\}$.

Thus the group $\rho_{p}\left(\left(P B_{3}\right)_{(k)}\right)$ contains $\rho_{p}\left(\left\langle g_{1}^{2}, g_{2}^{2}\right\rangle_{(k)}\right)$ and so it also contains a free non-abelian group. Therefore, Proposition 3.2 implies that $\rho_{p}\left(I_{g}(k)\right)$ contains a free non-abelian subgroup, which will complete the proof of Proposition 3.1 .

We further consider the remaining cases and briefly outline in section 3.2 the modifications needed to make the same strategy work also for small genus surfaces and for those values of the parameter $p$ which were excluded above, namely:

Proposition 3.4. Assume that $g$ and $p$ verify one of the following conditions:

1. $g=2, p$ is even and $p \notin\{4,8,12,16,24,40\}$;

2. $g=3$ and $p \notin\{3,4,8,12,16,24,40\}$;

3. $g \geq 4$ and $p=40$.

Then $\rho_{p}\left(M_{g}\right)$ contains a free non-abelian group.

Then Propositions 3.1 and 3.4 above will prove Theorem 1.1 .

\subsubsection{Proof of Proposition 3.2}

Choose the base point $*$ for the fundamental group $\pi_{1}\left(\Sigma_{g}\right)$ on the circle $c_{4}$ that separates the subsurfaces $\Sigma_{3,1}$ and $\Sigma_{g-3,1}$. Let $\varphi$ be a homeomorphism of $\Sigma_{0,4}$ that is identity on the boundary and whose mapping class $b$ belongs to $P B_{3} \subset M_{0,4}$. Consider its extension $\widetilde{\varphi}$ to $\Sigma_{g}$ by identity outside $\Sigma_{0,4}$. Its mapping class $B$ in $M_{g}$ is the image of $b$ in $M_{g}$.

In order to understand the action of $B$ on $\pi_{1}\left(\Sigma_{g}\right)$ we introduce three kinds of loops based at $*$ :

1. Loops of type I are those included in $\Sigma_{g-3,1}$.

2. Loops of type II are those contained in $\Sigma_{0,4}$.

3. Begin by fixing three simple arcs $\lambda_{1}, \lambda_{2}, \lambda_{3}$ embedded in $\Sigma_{0,4}$ joining $*$ to the three other boundary components $c_{1}, c_{2}$ and $c_{3}$, respectively. Loops of type III are of the form $\lambda_{i}^{-1} x \lambda_{i}$, where $x$ is some loop based at the endpoint of $\lambda_{i}$ and contained in the 1-holed torus bounded by $c_{i}$. Thus loops of type III generate $\pi_{1}\left(\Sigma_{3,1}, *\right)$.

Now, the action of $B$ on the homotopy classes of loops of type $\mathrm{I}$ is trivial. The action of $B$ on the homotopy classes of loops of type II is completely described by the action of $b \in P B_{3}$ on $\pi_{1}\left(\Sigma_{0,4}, *\right)$. Specifically, let $A: B_{3} \rightarrow \operatorname{Aut}\left(\mathbb{F}_{3}\right)$ be the Artin representation (see [1]). Here $\mathbb{F}_{3}$ is the free group on three generators $x_{1}, x_{2}, x_{3}$ which is identified with the fundamental group of the 3 -holed disk $\Sigma_{0,4}$.

Lemma 3.1. If $b \in\left(P B_{3}\right)_{(k)}$, then $A(b)\left(x_{i}\right)=l_{i}(b)^{-1} x_{i} l_{i}(b)$, where $l_{i}(b) \in\left(\mathbb{F}_{3}\right)_{(k)}$. 
Proof. This is folklore. Moreover, the statement is valid for any number $n$ of strands instead of 3. Here is a short proof avoiding heavy computations. It is known that the set $P B_{n, k}$ of those pure braids $b$ for which the length $m$ Milnor invariants of their Artin closures vanish for all $m \leq k$ is a normal subgroup $P B_{n, k}$ of $B_{n}$. Furthermore, the central series of subgroups $P B_{n, k}$ verifies the following (see e.g. [28]):

$$
\left[P B_{n, k}, P B_{n, m}\right] \subset P B_{n, k+m}, \text { for all } n, k, m,
$$

and hence, we have $\left(P B_{n}\right)_{(k)} \subset P B_{n, k}$.

Now, if $b$ is a pure braid, then $A(b)\left(x_{i}\right)=l_{i}(b)^{-1} x_{i} l_{i}(b)$, where $l_{i}(b)$ is the so-called longitude of the $i$-th strand. Next we can interpret Milnor invariants as coefficients of the Magnus expansion of the longitudes. In particular, this correspondence shows that $b \in P B_{n, k}$ if and only if $l_{i}(b) \in\left(\mathbb{F}_{n}\right)_{(k)}$. This proves the claim.

The action of $B$ on the homotopy classes of loops of type III can be described in a similar way. Let a homotopy class $a$ of this kind be represented by a loop $\lambda_{i}^{-1} x \lambda_{i}$. Then $\lambda_{i}^{-1} \varphi\left(\lambda_{i}\right)$ is a loop contained in $\Sigma_{0,4}$, whose homotopy class $\eta_{i}=\eta_{i}(b)$ depends only on $b$ and $\lambda_{i}$. Then it is easy to see that

$$
B(a)=\eta_{i}^{-1} a \eta_{i}
$$

Let now $y_{i}, z_{i}$ be standard homotopy classes of loops based at a point of $c_{i}$ which generate the fundamental group of the holed torus bounded by $c_{i}$, so that $\left\{y_{1}, z_{1}, y_{2}, z_{2}, y_{3}, z_{3}\right\}$ is a generator system for $\pi_{1}\left(\Sigma_{3,1}, *\right)$, which is the free group $\mathbb{F}_{6}$ of rank 6 .

Lemma 3.2. If $b \in\left(P B_{3}\right)_{(k)}$, then $\eta_{i}(b) \in\left(\mathbb{F}_{6}\right)_{(2 k)}$.

Proof. It suffices to observe that $\eta_{i}(b)$ is actually the $i$-th longitude $l_{i}(b)$ of the braid $b$, expressed now in the generators $y_{i}, z_{i}$ instead of the generators $x_{i}$. We also know that $x_{i}=\left[y_{i}, z_{i}\right]$. Let then $\eta: \mathbb{F}_{3} \rightarrow \mathbb{F}_{6}$ be the group homomorphism given on the generators by $\eta\left(x_{i}\right)=\left[y_{i}, z_{i}\right]$. Then $\eta_{i}(b)=\eta\left(l_{i}(b)\right)$. Eventually, if $l_{i}(b) \in\left(\mathbb{F}_{3}\right)_{(k)}$, then $\eta\left(l_{i}(b)\right) \in\left(\mathbb{F}_{6}\right)_{(2 k)}$ and the claim follows.

Therefore the class $B$ belongs to $I_{g}(k)$, since its action on every generator of $\pi_{1}\left(\Sigma_{g}, *\right)$ is a conjugation by an element of $\pi_{1}\left(\Sigma_{g}, *\right)_{(k)}$.

\subsubsection{Proof of Proposition 3.3}

First we want to identify some sub-representation of the restriction of $\rho_{p}$ to $P B_{3} \subset M_{g}$. Specifically we have:

Lemma 3.3. Let $p \geq 5$. The restriction of the quantum representation $\rho_{p}$ at $P B_{3} \subset M_{0,4}$ has an invariant 2-dimensional subspace such that the corresponding sub-representation is equivalent to the Burau representation $\beta_{-q_{p}}$, where the root of unity $q_{p}$ is given by:

$$
q_{p}= \begin{cases}-A_{p}^{-4}=-\exp \left(-\frac{8 \pi i}{p}\right), & \text { if } p \equiv 0(\bmod 4) \\ -A_{5}^{-4}=-\exp \left(-\frac{4 \pi i}{5}\right), & \text { if } p=5 ; \\ -A_{p}^{-8}=-\exp \left(-\frac{8(p+1) \pi i}{p}\right), & \text { if } p \equiv 1(\bmod 2), p \geq 7 .\end{cases}
$$

Proof. For even $p$ this is the content of [8], Prop. 3.2. We recall that in this case the invariant 2-dimensional subspace is the the space of conformal blocks associated to the surface $\Sigma_{0,4}$ with all boundary components being labeled by the color 1 . The odd case is similar. The invariant subspace is the space of conformal blocks associated to the surface $\Sigma_{0,4}$ with boundary labels $(2,2,2,2)$, when $p=5$ and $(4,2,2,2)$, when $p \geq 7$ respectively. The eigenvalues of the half-twist can be computed as in [8]. 
Thus the image $\rho_{p}\left(P B_{3}\right)$ of the quantum representation projects onto the image of the Burau representation $\beta_{-q_{p}}\left(P B_{3}\right)$.

Up to a Galois conjugacy we can assume that $\beta_{-q_{p}}$ is unitarizable and after rescaling, it takes values in $U(2)$. Consider the projection of $\beta_{-q_{p}}\left(\left(P B_{3}\right)_{(k)}\right)$ into $U(2) / U(1)=S O(3)$.

A finitely generated subgroup of $S O(3)$ is either finite or abelian or else dense in $S O(3)$. If the group is dense in $S O(3)$, then it contains a free non-abelian subgroup. Moreover, solvable subgroups of $S U(2)$ (and hence of $S O(3)$ ) are abelian. The finite subgroups of $S O(3)$ are well-known. They are the following: cyclic groups, dihedral groups, tetrahedral group (automorphisms of the regular tetrahedron), the octahedral group (the group of automorphisms of the regular octahedron) and the icosahedral group (the group of automorphisms of the regular icosahedron or dodecahedron). All but the last one are actually solvable groups. The icosahedral group is isomorphic to the alternating group $A_{5}$ and it is well-known that it is simple (and thus non-solvable). As a side remark this group appeared in relation with the non-solvability of the quintic equation in Felix Klein's monograph [18.

Lemma 3.4. If $q$ is not a primitive root of unity of order in the set $\{1,2,3,4,6,10\}$, then $\left(\Gamma_{q}\right)_{(k)}$ is non-solvable and thus non-abelian for any $k$. Moreover, $\left(\Gamma_{q}\right)_{(k)}$ cannot be $A_{5}$, for any $k$.

Proof. If $\left(\Gamma_{q}\right)_{(k)}$ were solvable, then $\Gamma_{q}$ would be solvable. But one knows that $\Gamma_{q}$ is not solvable. In fact if $q$ is as above, then $\Gamma_{q}$ is an infinite triangle group by Corollary 2.1.

Now any infinite triangle group has a finite index subgroup which is a surface group of genus at least 2. Therefore, each term of the lower central series of that surface group embeds into the corresponding term of the lower central series of $\Gamma_{q}$, so that the later is non-trivial. Since the lower central series of a surface group of genus at least 2 consists only of infinite groups it follows that no term can be isomorphic to the finite group $A_{5}$ either.

Lemma 3.4 shows that whenever $p$ is as in the statement of Proposition 3.3 , the group $\beta_{-q_{p}}\left(\left(\left\langle g_{1}^{2}, g_{2}^{2}\right\rangle\right){ }_{(k)}\right)$ is neither finite nor abelian, so that it is dense in $S O(3)$ and hence it contains a free non-abelian group. This proves Proposition 3.3 .

\subsubsection{Explicit free subgroups}

The main interest of the elementary arguments in the proof presented above is that the free nonabelian subgroups in the image are abundant and explicit. For instance we have:

Theorem 3.1. Assume that $g \geq 4, p \notin\{3,4,12,16\}$ and $p \not \equiv 8(\bmod 16)$. Set $x=\rho_{p}\left(\left[g_{1}^{2}, g_{2}^{2}\right]\right)$ and $y=\rho_{p}\left(\left[g_{1}^{4}, g_{2}^{2}\right]\right)$. Then the group generated by the iterated commutators $[x,[x,[x, \ldots,[x, y]] \ldots]$ and $\left[y,[x,[x, \ldots,[x, y]] \ldots]\right.$ of length $k \geq 3$ is a free non-abelian subgroup of $\rho_{p}\left(I_{g}(k)\right)$.

It is well-known that the order of the matrix $\beta_{-q}\left(g_{i}\right), i \in\{1,2\}$ in $P G L(2, \mathbb{C})$ is the order of the root of unity $q$, namely the smallest positive $n$ such that $q$ is a primitive root of unity of order $n$.

We considered in Lemma 3.3 the root of unity $q_{p}$ with the property that $\beta_{-q_{p}}$ is a sub-representation of the quantum representation $\rho_{p}$. We derive from Lemma 3.3 that the order of the root of unity $q_{p}$ is $2 o(p)$ where

$$
o(p)= \begin{cases}\frac{p}{4}, & \text { if } p \equiv 4(\bmod 8) \\ \frac{p}{8}, & \text { if } p \equiv 0(\bmod 16) \\ \frac{p}{16}, & \text { if } p \equiv 8(\bmod 16) \\ p, & \text { if } p \equiv 1(\bmod 2), p \geq 5 .\end{cases}
$$

Therefore $\beta_{-q_{p}}\left(\left\langle g_{1}^{2}, g_{2}^{2}\right\rangle\right)$ is isomorphic to the triangle group $\Delta(o(p), o(p), o(p))$. Notice that in general $o(p) \in \frac{1}{2}+\mathbb{Z}$ and $o(p)$ is an integer if and only if $p \not \equiv 8(\bmod 16)$, as we suppose from now on. 
Observe also that the order of $\beta_{-q_{p}}\left(g_{1}^{2}\right)$ is a proper divisor of the order $p$ of a Dehn twist $\rho_{p}\left(g_{1}^{2}\right)$, when $p$ is even.

In the proof of Theorem 3.1 we will need the following result concerning the structure of commutator subgroups of triangle groups:

Lemma 3.5. The commutator subgroup $\Delta(r, r, r)_{(2)}$ of a triangle group $\Delta(r, r, r), r \in \mathbb{Z}-\{0,1,2\}$, is a 1-relator group with generators $\widetilde{c_{i j}}$, for $1 \leq i, j \leq r-1$, and the relation:

$$
\widetilde{c_{11}} \cdot{\widetilde{c_{21}}}^{-1} \cdot \widetilde{c_{22}} \cdot \widetilde{c_{32}}-1,{\widetilde{c_{r r-1}}}^{-1} \cdot \widetilde{c_{r r}}=1
$$

Proof. The kernel $K$ of the abelianization homomorphism $\mathbb{Z} / r \mathbb{Z} * \mathbb{Z} / r \mathbb{Z} \rightarrow \mathbb{Z} / r \mathbb{Z} \times \mathbb{Z} / r \mathbb{Z}$ is the free group generated by the commutators. Denote by $\widetilde{a}$ and $\widetilde{b}$ the generators of the two copies of the cyclic group $\mathbb{Z} / r \mathbb{Z}$. Then $K$ is freely generated by $\widetilde{c_{i j}}=\left[\widetilde{a}^{i}, \widetilde{b}^{j}\right]$, where $1 \leq i, j \leq r-1$. The group $\Delta(r, r, r)$ is the quotient of $\mathbb{Z} / r \mathbb{Z} * \mathbb{Z} / r \mathbb{Z}$ by the normal subgroup generated by the element $(\widetilde{a} \widetilde{b})^{r} \widetilde{a}^{-r} \widetilde{b}^{-r}$, which belongs to $K$. This shows that $\Delta(r, r, r)_{(2)}$ is a 1-relator group, namely the quotient of $K$ by the normal subgroup generated by the element $(\widetilde{a} \tilde{b})^{r} \widetilde{a}^{-r} \widetilde{b}^{-r}$. In order to get the explicit form of the relation we have to express this element as a product of the generators of $K$, i.e., as a product of commutators of the form $\left[\widetilde{a}^{i}, \widetilde{b}^{j}\right]$. This can be done as follows:

$$
(\widetilde{a} \widetilde{b})^{r} \widetilde{a}^{-r} \widetilde{b}^{-r}=[\widetilde{a}, \widetilde{b}]\left[\widetilde{b}, \widetilde{a}^{2}\right]\left[\widetilde{a}^{2}, \widetilde{b}^{2}\right] \cdots\left[\widetilde{a}^{r-1}, \widetilde{b}^{r-1}\right]\left[\widetilde{b}^{r-1}, \widetilde{a}^{r}\right]\left[\widetilde{a}^{r}, \widetilde{b}^{r}\right]
$$

Therefore $\Delta(r, r, r)_{(2)}$ has a presentation with generators $\widetilde{c_{i j}}$, where $1 \leq i \leq j \leq r$, and the relation in the statement of the lemma.

Proof of Theorem 3.1. Recall now the classical Magnus Freiheitsatz, which states that any subgroup of a 1-relator group which is generated by a proper subset of the set of generators involved in the cyclically reduced word relator is free.

Assume now that $o(p) \in \mathbb{Z}$ and $o(p) \geq 4$. Then $\beta_{-q_{p}}\left(\left[g_{1}^{2}, g_{2}^{2}\right]\right)$ and $\beta_{-q_{p}}\left(\left[g_{1}^{4}, g_{2}^{2}\right]\right)$ are the elements $\widetilde{c_{11}}$ and $\widetilde{c_{21}}$ of $\Delta(o(p), o(p), o(p))_{(2)}$ respectively.

An easy application of the Freiheitsatz to the commutator subgroup of the infinite triangle group $\Delta(o(p), o(p), o(p))$ gives us that the subgroup generated by $\beta_{-q_{p}}\left(\left[g_{1}^{2}, g_{2}^{2}\right]\right)$ and $\beta_{-q_{p}}\left(\left[g_{1}^{4}, g_{2}^{2}\right]\right)$ is free. This implies that the subgroup generated by $x$ and $y$ is free.

Eventually the $k$-th term of the lower central series of the group generated by $x$ and $y$ is also a free subgroup which is contained into $\rho_{p}\left(\left(P B_{3}\right)_{(k)}\right) \subset \rho_{p}\left(I_{g}(k)\right)$. This proves Theorem 3.1.

When $p \equiv 8(\bmod 16), o(p)$ is a half-integer and $\beta_{-q_{p}}\left(\left\langle g_{1}^{2}, g_{2}^{2}\right\rangle\right)$ is isomorphic to the triangle group $\Delta(2,3,2 o(p))$. If $\operatorname{gcd}(3,2 o(p))=1$, then $H_{1}(\Delta(2,3,2 o(p)))=0$, so the central series of this triangle group is trivial. Nevertheless the group $\Delta(2,3,2 o(p))$ has many normal subgroups of finite index which are surface groups and thus contain free subgroups. In particular, any subgroup of infinite index of $\Delta(2,3,2 o(p))$ is free. There is then an extension of the previous result in this case, as follows:

Theorem 3.2. Assume that $g \geq 4, p \notin\{8,24,40\}$ and $p \equiv 8(\bmod 16)$ so that $p=8 n$, for odd $n=2 k+1 \geq 7$. Consider the following two elements of $\left\langle g_{1}^{2}, g_{2}^{2}\right\rangle$ :

$$
s=g_{1}^{2 k} g_{2}^{2 k} g_{1}^{2\left(k-k^{2}\right)} g_{2}^{-2} g_{1}^{2 k} g_{2}^{-2} g_{1}^{2\left(k-k^{2}\right)} g_{2}^{2 k} g_{1}^{2 k}
$$

and

$$
t=g_{1}^{2 k} g_{2}^{2 k} g_{1}^{2\left(k-k^{2}\right)} g_{2}^{-2} g_{1}^{2(k+1)} g_{2}^{2} g_{1}^{2\left(k+k^{2}\right)} g_{2}^{2 k} g_{1}^{10 k}
$$


Let $N(s, t)$ be the normal subgroup generated by $s$ and $t$ in $\left\langle g_{1}^{2}, g_{2}^{2}\right\rangle$. Then for any choice of $f(n)$ elements $x_{1}, x_{2}, \ldots, x_{f(n)}$ from $N(s, t)$ the image $\rho_{p}\left(\left\langle x_{1}, x_{2}, \ldots, x_{f(n)}\right\rangle\right)$ is a free group. Here the function $f(n)$ is given by:

$$
f(n)=|P S L(2, \mathbb{Z} / n \mathbb{Z})| \cdot \frac{n-6}{6 n}
$$

and, in particular, when $n$ is prime by:

$$
f(n)=\frac{(n+1)(n-1)(n-6)}{12} .
$$

Then the group generated by the iterated commutators of length $k \geq 3$ is a free subgroup of $\rho_{p}\left(I_{g}(k)\right)$.

Proof. Observe that the map $P S L(2, \mathbb{Z}) \rightarrow P S L(2, \mathbb{Z} / n \mathbb{Z})$ factors through $\Delta(2,3, n)$, namely we have a homomorphism $\psi: \Delta(2,3, n) \rightarrow P S L(2, \mathbb{Z} / n \mathbb{Z})$ defined by

$$
\psi(\alpha)=\left(\begin{array}{cc}
1 & -1 \\
0 & 1
\end{array}\right), \psi(u)=\left(\begin{array}{cc}
1 & -1 \\
1 & 0
\end{array}\right), \psi(v)=\left(\begin{array}{cc}
0 & -1 \\
1 & 0
\end{array}\right) .
$$

The matrices $\psi(\alpha), \psi(u), \psi(v)$ are obviously elements of orders $n, 3$ and 2 in $P S L(2, \mathbb{Z} / n \mathbb{Z})$ respectively. It follows that the normal subgroup $K(2,3, n)=\operatorname{ker} \psi$ is torsion free, because every torsion element in $\Delta(2,3, n)$ is conjugate to some power of one the generators $\alpha, u, v$ (see [13]). Therefore $K(2,3, n)$ is a surface group, namely the fundamental group of a closed orientable surface which finitely covers the fundamental domain of $\Delta(2,3, n)$. The Euler characteristic $\chi(K(2,3, n))$ of this Fuchsian group can easily be computed by means of the formula:

$$
\chi(K(2,3, n))=|P S L(2, \mathbb{Z} / n \mathbb{Z})| \cdot \chi(\Delta(2,3, n)),
$$

where the (orbifold) Euler characteristic $\chi(\Delta(2,3, n))$ has the well-known expression:

$$
-\chi(\Delta(2,3, n))=1-\left(\frac{1}{2}+\frac{1}{3}+\frac{1}{n}\right)=\frac{n-6}{6 n} .
$$

It is also known that any $-\chi(G)+1$ elements of a closed orientable surface group $G$ generate a free subgroup of $G$. Thus, in order to establish Theorem 3.2, it will suffice to show that the images of the elements $s, t$ under Burau's representation $\beta_{-q_{p}}$ are normal generators of the group $K(2,3, n)$. This is equivalent to show that these images correspond to the relations needed to impose in $\Delta(2,3, n)$ in order to obtain the quotient $P S L(2, \mathbb{Z} / n \mathbb{Z})$. However, one already knows presentations for this group (see 4], Lemma 1 and [14]) as follows:

$$
P S L(2, \mathbb{Z} / n \mathbb{Z})=\left\langle\alpha, v, u \mid \alpha^{n}=u^{3}=v^{2}=1, g v g v=g \alpha g^{-1} \alpha^{-4}=1\right\rangle,
$$

for odd $n$, where $g=v \alpha^{k} v \alpha^{-2} v \alpha^{k}$. The first three relations above correspond to the presentation of $\Delta(2,3, n)$ and the elements $g v g v$ and $g \alpha g \alpha^{-4}$ correspond to the images of $s$ and $t$ in $\Delta(2,3, n)$, by using the fact that $\alpha=a^{k+1}, v \alpha^{2} v=b, v=a^{k} b^{k} a^{k}$ (see the proof of Lemma 2.1).

\subsubsection{Second proof of Proposition 3.1}

We outline here an alternative proof which does not rely on the description of the image of $\mathrm{Bu}-$ rau's representation in Corollary 2.1. This proof is shorter but less effective since it does not produce explicit free subgroups and uses the result of [21] and the Tits alternative, which need more sophisticated tools from the theory of algebraic groups.

The image $\rho_{p}\left(M_{g}\right)$ in $P U(N(p, g))$ is dense in $P S U(N(p, g))$, if $p \geq 5$ is prime (see [21]), where $N(p, g)$ denotes the dimension of the space of conformal blocks in genus $g$ for the TQFT $\mathcal{V}_{p}$. In 
particular, the image of $\rho_{p}$ is Zariski dense in $P U(N(p, g))$. By the Tits alternative (see [30]) the image is either solvable or else it contains a free non-abelian subgroup. However, if the image were solvable, then its Zariski closure would be a solvable Lie group, which is a contradiction. This implies that $\rho_{p}\left(M_{g}\right)$ contains a free non-abelian subgroup.

If $p$ is not prime but has a prime factor $r \geq 5$, then the claim for $p$ follows from that for $r$. If $p$ does not satisfy this condition, then we have again to use the result of Proposition 3.3 for $k=1$. This result can be obtained directly from the computations in [17] proving that the image of the Jones representation of $B_{3}$ is neither finite nor abelian for the considered values of $p$. This settles the case $k=1$ of Proposition 3.1.

Furthermore, the group $\rho_{p}\left(T_{g}\right)$ is of finite index in $\rho_{p}\left(M_{g}\right)$, and hence it also contains a free nonabelian subgroup. Results of Morita (see [27]) show that for $k \geq 2$ the group $I_{g}(k+1)$ is the kernel of the $k$-th Johnson homomorphism $I_{g}(k) \rightarrow A_{k}$, where $A_{k}$ is a finitely generated abelian group. This implies that $\left[I_{g}(k), I_{g}(k)\right] \subset I_{g}(k+1)$, for every $k \geq 2$. In particular, the $k$-th term of the derived series of $\rho_{p}\left(T_{g}\right)$ is contained into $\rho_{p}\left(I_{g}(k+1)\right)$. But every term of the derived series of $\rho_{p}\left(T_{g}\right)$ contains the corresponding term of the derived series of a free subgroup and hence a free non-abelian group. This proves Proposition 3.1 .

Remark 3.1. Using the strong version of Tits' theorem due to Breuillard and Gelander (see [3]) there exists a free non-abelian subgroup of $M_{g} / M_{g}[p]$ whose image in $P S U(N(p, g))$ is dense. Here $M_{g}[p]$ denotes the (normal) subgroup generated by the $p$-th powers of Dehn twists.

\subsection{Proof of Proposition 3.4}

If the genus $g \in\{2,3\}$, then the construction used in the proof of Proposition 3.1 should be modified. This is equally valid when we want to get rid of the values $p=5$ and $p=40$.

The proof follows along the same lines as Proposition 3.3, but the embeddings $\Sigma_{0,4} \subset \Sigma_{g}$ are now different. In all cases considered below the analogue of Proposition 3.2 will still be true, namely the image of the subgroup $\left\langle g_{1}^{2}, g_{2}^{2}\right\rangle_{(k)}$ by the homomorphisms $M_{0,4} \rightarrow M_{g}$ will be contained within the Johnson subgroup $I_{g}(k)$.

If $g=2$ we use the following embedding $\Sigma_{0,4} \subset \Sigma_{2}$ :

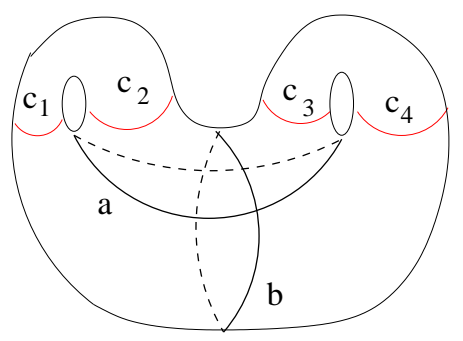

Although the homomorphism $M_{0,4} \rightarrow M_{2}$ induced by this embedding is not anymore injective, it sends the free subgroup $\left\langle g_{1}^{2}, g_{2}^{2}\right\rangle \subset P B_{3} \subset M_{0,4}$ isomorphically onto the subgroup of $M_{2}$ generated by the Dehn twists along the curves $a$ and $b$ in the figure above.

Consider for even $p$ the space of conformal blocks associated to $\Sigma_{0,4}$ with boundary labels $(1,1,1,1)$. This 2-dimensional subspace is $\rho_{p}\left(\left\langle g_{1}^{2}, g_{2}^{2}\right\rangle\right)$-invariant and the restriction of $\rho_{p}$ to this subspace is still equivalent to Burau's representation $\beta_{-q_{p}}$ (see [8]). Therefore Proposition 3.3 shows that $\rho_{p}\left(\left\langle g_{1}^{2}, g_{2}^{2}\right\rangle_{(k)}\right)$, and hence also $\rho_{p}\left(I_{2}(k)\right)$, contains a free non-abelian group.

If $g=3$ and $p \geq 7$ is odd, then we consider the following embedding of $\Sigma_{0,4} \subset M_{3}$ : 


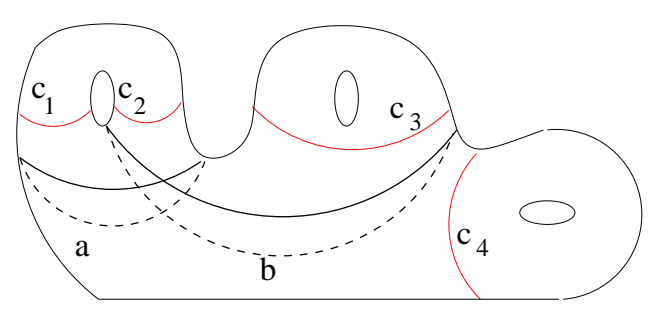

The homomorphism $M_{0,4} \rightarrow M_{3}$ induced by this embedding is not injective but it also sends the free subgroup $\left\langle g_{1}^{2}, g_{2}^{2}\right\rangle \subset P B_{3} \subset M_{0,4}$ isomorphically onto the subgroup of $M_{3}$ generated by the Dehn twists along the curves $a$ and $b$ in the figure above. The space of conformal blocks associated to $\Sigma_{0,4}$ with boundary labels $(2,2,2,4)$ is a 2 -dimensional subspace invariant by $\rho_{p}\left(\left\langle g_{1}^{2}, g_{2}^{2}\right\rangle\right)$ and the restriction of $\rho_{p}$ to this subspace is equivalent to Burau's representation $\beta_{-q_{p}}$. Applying again Proposition 3.3, we find that $\rho_{p}\left(\left\langle g_{1}^{2}, g_{2}^{2}\right\rangle_{(k)}\right)$, and hence $\rho_{p}\left(I_{3}(k)\right)$, contains a free non-abelian group. This also gives the desired results for any $g \geq 3$, and $p$ as in the statement.

Eventually we have to settle the case $p=40$, when $\beta_{-q_{p}}\left(B_{3}\right)$ is known to have finite image (see [17]). We will consider instead the representation $\rho_{p}\left(i\left(P B_{4}\right)\right)$, where $P B_{4}$ embeds non-canonically into $M_{0,5}$ and $M_{0,5}$ maps into $M_{3}$ by the homomorphism $i: M_{0,5} \rightarrow M_{3}$ induced by the inclusion $\Sigma_{0,5} \subset \Sigma_{g}$ drawn below:

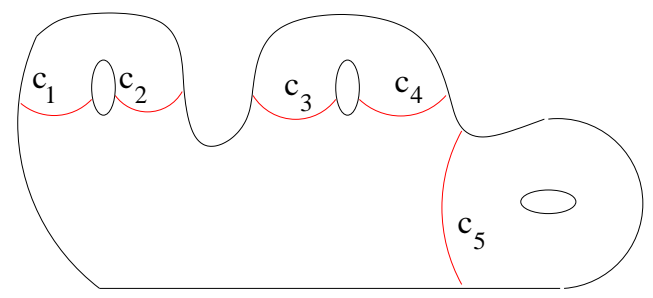

We consider the 3-dimensional space of conformal blocks associated to the surface $\Sigma_{0,5}$ with the boundary labels $(1,1,1,1,2)$, when $p=40$ and the labels $(2,2,2,2,2)$, when $p=5$ respectively. This space of conformal blocks is $\rho_{p}\left(i\left(P B_{4}\right)\right)$-invariant. The restriction of $\left.\rho_{p}\right|_{P B_{4}}$ to this invariant subspace is known (see again [8]) to be equivalent to the Jones representation of $B_{4}$ at the corresponding root of unity.

Now we have to use a result of Freedman, Larsen and Wang (see[7]) subsequently reproved and extended by Kuperberg in ([20], Thm.1) saying that the Jones representation of $B_{4}$ at a 10-th root of unity on the two 3-dimensional conformal blocks we chose is Zariski dense in the group $S L(3, \mathbb{C})$. A particular case of the Tits alternative says that any finitely generated subgroup of $S L(3, \mathbb{C})$ is either solvable or else contains a free non-abelian group. A solvable subgroup has also a solvable Zariski closure. The denseness result from above implies then that $\rho_{p}\left(P B_{4}\right)$, and hence also $\rho_{p}\left(\left(P B_{4}\right)_{(k)}\right)$, contains a free non-abelian group. The arguments in the proof of Proposition 3.3 carry on to this setting and this proves Proposition 3.4 .

Corollary 3.3. For any $k$ the quotient group $I_{g}(k) / M_{g}[p] \cap I_{g}(k)$, and in particular, $K_{g} / K_{g}[p]$, for $g \geq 3$ and $p \notin\{3,4,8,12,16,24\}$ contains a free non-abelian subgroup. Here $K_{g}[p]$ is the normal subgroup of $K_{g}$ generated by the $p$-th powers of the Dehn twist along separating simple closed curves.

\section{References}

[1] J. Birman, Braids, Links, and Mapping Class Groups, Ann. Math. Studies, Princeton Univ. Press, 1969.

[2] C. Blanchet, N. Habegger, G. Masbaum and P. Vogel, Topological quantum field theories derived from the Kauffman bracket, Topology 34(1995), 883927. 
[3] E. Breuillard and T. Gelander, On dense free subgroups of Lie groups, J. Algebra 261(2003), 448-467.

[4] A. Coste and T. Gannon, Congruence subgroups and rational conformal field theory, arxiv:math/9909080

[5] M. Deraux, On the universal cover of certain exotic Kähler surfaces of negative curvature, Math. Annalen 329(2004), 653-683.

[6] A. Dimca and S. Papadima, Arithmetic group symmetry and finiteness properties of Torelli groups, arXiv:1002.0673.

[7] M. H. Freedman, M. Larsen and Z. Wang, The two-eigenvalue problem and density of Jones representation of braid groups, Comm. Math. Phys. 228(2002), 177199.

[8] L. Funar, On the TQFT representations of the mapping class groups, Pacific J. Math. 188(2)(1999), 251-274.

[9] L. Funar, On power subgroups of mapping class groups, 15p., arXiv:0910.1493.

[10] L. Funar and T. Kohno, On Burau representations at roots of unity, 18p., arXiv:0907.0568.

[11] S. Garoufalidis and J. Levine, Finite type 3-manifold invariants and the structure of the Torelli group. I, Invent. Math. 131(1998), 541-594.

[12] P. Gilmer and G. Masbaum, Integral lattices in TQFT, Ann. Sci. Ecole Norm. Sup. (4) 40(2007), 815844.

[13] H. M. A. Hoare, A. Karrass and D. Solitar, Subgroups of infinite index in Fuchsian groups, Math. Zeitschrift 125(1972), 5969 .

[14] J. Hurrelbrink, On presentations of $S L_{n}\left(\mathbb{Z}_{s}\right)$, Commun. Algebra 11(1983), 937-947.

[15] D. L. Johnson, Homeomorphisms of a surface which act trivially on homology, Proc. Amer. Math. Soc. 75(1979), 119125.

[16] D. L. Johnson, The structure of the Torelli group. II. A characterization of the group generated by twists on bounding curves, Topology 24(1985), 113-126.

[17] V. F. R. Jones, Braid groups, Hecke algebras and type $I I_{1}$ factors, in Geometric methods in operator algebras, Proc. US-Japan Seminar, (H. Araki, E. G. Effros Eds.), 1986, 242-271.

[18] F. Klein, Lectures on the icosahedron and the solution of equation of the fifth degree, Dover Publications, 2nd edition (1956) originally published in 1884.

[19] T. Kohno, Topological invariants for 3-manifolds using representations of mapping class groups I, Topology, 31 (1992), 203-230.

[20] G. Kuperberg, Denseness and Zariski denseness of Jones braid representations, Geometry \& Topology 15 (2011), 11-39.

[21] M. Larsen and Zhenghan Wang, Density of the SO(3) TQFT representation of mapping class groups, Comm. Math. Phys. 260(2005), 641-658.

[22] J. Levine, Pure braids, a new subgroup of the mapping class group and finite-type invariants, Tel Aviv Topology Conference: Rothenberg Festschrift (1998), 137-157, Contemp. Math., 231, Amer. Math. Soc., Providence, RI, 1999.

[23] G. Masbaum, An element of infinite order in TQFT-representations of mapping class groups, Lowdimensional topology (Funchal, 1998), 137-139, Contemp. Math., 233, Amer. Math. Soc., Providence, RI, 1999.

[24] G. Masbaum, On representations of mapping class groups in integral TQFT, Oberwolfach Reports, Vol. 5, issue 2, 2008, pp. 1202-1205.

[25] C. T. McMullen, Braid groups and Hodge theory, 2009, Math. Annalen, to appear.

[26] S. Morita, On the structure of the Torelli group and the Casson invariant, Topology 30(1991), 603-621. 
[27] S. Morita, Abelian quotients of subgroups of the mapping class group of surfaces, Duke Math. J. 70(1993), 699-726.

[28] T. Ohkawa, The pure braid groups and the Milnor $\bar{\mu}$-invariants of links, Hiroshima Math. J. 12(1982), 485-489.

[29] N. Y. Reshetikhin and V. G. Turaev, Invariants of 3-manifolds via link polynomials and quantum groups, Invent. Math., 103 (1991), 547-597.

[30] J. Tits, Free subgroups in linear groups, J. Algebra 20(1972), 250-270. 